\title{
EXISTENCE OF BEST $n$-CONVEX APPROXIMATIONS
}

\author{
D. ZWICK
}

\begin{abstract}
We prove that every function $f$, continuous on a compact interval $[a, b]$, has a continuous, best $n$-convex approximation with respect to the uniform norm on $[a, b]$.
\end{abstract}

Introduction. A real-valued function $g$ defined on a real interval $I$ is called $n$-convex if its $n$th order divided differences $\left[x_{0}, \ldots, x_{n}\right] g$ are nonnegative for all distinct $x_{0}, \ldots, x_{n}$ in $I$, or equivalently if the 'augmented Vandermonde'

$$
\left|\begin{array}{ccc}
1 & \cdots & 1 \\
x_{0} & \cdots & x_{n} \\
\vdots & & \vdots \\
x_{0}^{n-1} & \cdots & x_{n}^{n-1} \\
g\left(x_{0}\right) & \cdots & g\left(x_{n}\right)
\end{array}\right|
$$

is nonnegative for all $x_{0}<\cdots<x_{n}$ in $I$. Thus a 1-convex function is increasing and a 2-convex function is convex in the usual sense. $n$-convex functions need not be $n$-times differentiable, however if $g^{(n)}$ is continuous, then $g$ is $n$-convex iff $g^{(n)} \geq 0$.

For $f \in C[a, b]$, an $n$-convex function $g$ is a best $n$-convex approximation to $f$ if

$$
\|f-g\|=\inf \{\|f-\tilde{g}\|: \tilde{g} \text { is } n \text {-convex on }[a, b]\},
$$

where $\|\cdot\|$ denotes the supremum norm on $[a, b]$. As noted below, for $n \geq 2$, any function that is $n$-convex on $[a, b]$ is continuous and bounded in $(a, b)$ so that if a best $n$-convex approximation exists then a continuous, best $n$-convex approximation exists as well.

Although the subject of best $n$-convex approximation has been treated in a number of papers over the past fifteen years (usually as 'monotone' or 'restricted derivative' approximation), these have dealt almost exclusively with $n$-convex approximation by polynomials $[\mathbf{3}, \mathbf{4}]$, for which an extensive theory now exists. Only recently have authors even begun to consider approximation by monotone functions in general [1] or by convex functions [2]. Except for the monotone case $n=1$, existence of continuous, best $n$-convex approximations to continuous functions has yet to be demonstrated. This is the purpose of this paper. ${ }^{1}$

Main results. As the case $n=1$ has been thoroughly treated in [1], it will be tacitly assumed hereafter that $n \geq 2$.

The first lemma follows directly from [8, Lemma 1.1].

Received by the editors March 19, 1985.

1980 Mathematics Subject Classification. Primary 41A50, 41A29; Secondary 26A51.

${ }^{1}$ The author has recently discovered that a similar result was proved by H. G. Burchard in his dissertation, Interpolation and approximation by generalized convex functions, Purdue, 19678. Burchard's approach is different and somewhat more complicated than the one taken here. 
(1) LEMMA. Let $F$ be a collection of $n$-convex functions, defined on $[a, b]$ and pointwise bounded on a dense subset of $[a, b]$. Then $F$ is uniformly bounded on compact subsets of $(a, b)$.

This result, and the following theorem, generalize a similar result for convex functions (see e.g. [5, p. 167]).

(2) Definition. A family $F$ of real-valued functions defined on $I \subset R$ is said to be uniformly Lipschitz-continuous if there is an $L<\infty$ such that $|f(x)-f(y)|<$ $L|x-y|$ for all $x, y \in I$ and all $f \in F$.

(3) THEOREM. Let $\left\{f_{k}\right\}$ be a sequence of $n$-convex functions defined on $(a, b)$. If $\left\{f_{k}\right\}$ is uniformly bounded on compact subsets of $(a, b)$ then it is uniformly Lipschitzcontinuous on compact subsets of $(a, b)$.

PROOF. Suppose that $\left\{f_{k}\right\}$ is not uniformly Lipschitz-continuous on $[c, d] \subset$ $(a, b)$. Then there is a subsequence, which we relabel as $\left\{f_{k}\right\}$, and sequences $\left\{x_{k}\right\}$ and $\left\{y_{k}\right\}$ with the properties $c \leq x_{k}<y_{k} \leq d, x_{k} \rightarrow x$ and $y_{k} \rightarrow x \in[c, d]$, and

$$
\left|\frac{f_{k}\left(y_{k}\right)-f_{k}\left(x_{k}\right)}{y_{k}-x_{k}}\right| \rightarrow \infty .
$$

Without loss of generality the sequence of difference quotients

$$
\frac{f_{k}\left(y_{k}\right)-f_{k}\left(x_{k}\right)}{y_{k}-x_{k}}
$$

converges either to $+\infty$ or to $-\infty$. In the latter case, we choose points $a<t_{0}<$ $\cdots<t_{n-2}<x$, and suppose that $k$ is large enough so that $t_{n-2}<x_{k}<y_{k}$. Then, by the $n$-convexity of $f_{k}$,

$$
D_{k}=\left|\begin{array}{ccccc}
1 & \cdots & 1 & 1 & 1 \\
t_{0} & \cdots & t_{n-2} & x_{k} & y_{k} \\
\vdots & & \vdots & \vdots & \vdots \\
t_{0}^{n-1} & \cdots & t_{n-2}^{n-1} & x_{k}^{n-1} & y_{k}^{n-1} \\
f_{k}\left(t_{0}\right) & \cdots & f_{k}\left(t_{n-2}\right) & f_{k}\left(x_{k}\right) & f_{k}\left(y_{k}\right)
\end{array}\right| \geq 0
$$

This determinant is unchanged if we subtract from each column but the first its predecessor, and then divide every element in a column (for each but the first column) by the element in its second row. We are then left with

$$
D_{k}=c_{k} \cdot\left|\begin{array}{cccc}
1 & \cdots & 1 & 1 \\
{\left[t_{0}, t_{1}\right] t^{2}} & \cdots & {\left[t_{n-2}, x_{k}\right] t^{2}} & {\left[x_{k}, y_{k}\right] t^{2}} \\
\vdots & & \vdots & \vdots \\
{\left[t_{0}, t_{1}\right] t^{n-1}} & \cdots & {\left[t_{n-2}, x_{k}\right] t^{n-1}} & {\left[x_{k}, y_{k}\right] t^{n-1}} \\
{\left[t_{0}, t_{1}\right] f_{k}} & \cdots & {\left[t_{n-2}, x_{k}\right] f_{k}} & {\left[x_{k}, y_{k}\right] f_{k}}
\end{array}\right|
$$

where $c_{k}=\prod_{i=0}^{n-3}\left(t_{i+1}-t_{i}\right) \cdot\left(x_{k}-t_{n-2}\right) \cdot\left(y_{k}-x_{k}\right)>0$, and $[x, y] f$ is the usual difference quotient. We thus have $D_{k} / c_{k} \geq 0$ for large enough $k$. If we now expand $D_{k} / c_{k}$ and use the uniform boundedness of $\left\{f_{k}\right\}$ we get

$$
0 \leq D_{k} / c_{k}=\left[x_{k}, y_{k}\right] f_{k} \cdot V_{k}+O(1)
$$


where $\left\{V_{k}\right\}$ is a sequence of determinants converging to a positive multiple of the Vandermonde determinant $V\left(t_{0}, \ldots, t_{n-2}, x\right)>0$. Since by assumption $\left[x_{k}, y_{k}\right] f_{k}$ $\rightarrow-\infty$ we have arrived at a contradiction.

If the difference quotients $(*)$ converge to $+\infty$ then we choose points $a<t_{0}<$ $\cdots<t_{n-3}<x \leq d<t_{n}<b$ and proceed as in the previous case. Here, for large enough $k, 0 \leq D_{k} / c_{k}=-\left[x_{k}, y_{k}\right] f_{k} \cdot V_{k}+O(1)$, where the $V_{k}$ 's converge to a positive multiple of the Vandermonde $V\left(t_{0}, \ldots, t_{n-3}, x, t_{n}\right)$. Again we arrive at a contradiction and hence $\left\{f_{k}\right\}$ must be uniformly Lipschitz-continuous on $[c, d]$.

By setting $f_{k} \equiv f$ for each $k$ in the proof of (3) we get

(4) CoRollary. If $f$ is $n$-convex on $[a, b]$ then $f$ is Lipschitz-continuous on compact subsets of $(a, b)$.

The following theorem extends an important convergence result, known to hold for convex functions $[\mathbf{5}, \mathbf{6}]$, to $n$-convex functions in general.

(5) THEOREM. Let $\left\{f_{k}\right\}$ be a sequence of $n$-convex functions, pointwise bounded on a dense subset of $(a, b)$. Then there is an $n$-convex function $f$ such that $a$ subsequence of $\left\{f_{k}\right\}$ converges uniformly to $f$ and compact subsets of $(a, b)$.

PROOF. By (1) $\left\{f_{k}\right\}$ is uniformly bounded on compact subsets of $(a, b)$, hence by (3) it is uniformly Lipschitz-continuous, and thus is equicontinuous, on compact subsets of $(a, b)$. By a corollary to the Arzela-Ascoli theorem [7, p. 179] a subsequence of $\left\{f_{k}\right\}$ converges pointwise to a continuous function $f$, which is therefore $n$-convex, and the convergence is uniform on compact subsets of $(a, b)$.

We now prove the main result of this paper.

(6) THEOREM. Every function $f \in C[a, b]$ has a best $n$-convex approximation $g \in C[a, b]$.

ProOF. We note first that if $n \geq 2$ then an $n$-convex function $g$ defined on $[a, b]$ is continuous in $(a, b)$ and bounded [6], and thus if $f \in C[a, b]$ then

$$
\sup _{[a, b]}|f(x)-g(x)| \geq \sup _{(a, b)}|f(x)-g(x)|=\sup _{[a, b]}\left|f(x)-g^{*}(x)\right|,
$$

where $g^{*}$ is the continuous $n$-convex function that agrees with $g$ in $(a, b)$ and satisfies $g^{*}(a)=g(a+), g^{*}(b)=g(b-)$. Thus we may, and will, restrict our attention in this proof to continuous $n$-convex functions.

Choose a sequence of $n$-convex functions $\left\{g_{k}\right\}$ such that

$$
\left\|f-g_{k}\right\| \downarrow E(f)=\inf \{\|f-\tilde{g}\|: \tilde{g} \text { is } n \text {-convex on }[a, b]\} \text {. }
$$

Since $\left\|g_{k}\right\|-\|f\| \leq\left\|f-g_{k}\right\| \leq\left\|f-g_{1}\right\|$, we have $\left\|g_{k}\right\| \leq\|f\|+\left\|f-g_{1}\right\|$ for all $k$, hence $\left\{g_{k}\right\}$ is uniformly bounded.

By (5) there is a subsequence, which we relabel $\left\{g_{k}\right\}$, and an $n$-convex function $g$ such that $g_{k} \rightarrow g$ uniformly on compact subsets of $(a, b)$. Moreover, $g$ is bounded and, as noted above, may be extended to a continuous function on $[a, b]$.

We now show that $g$ is a best $n$-convex approximation to $f$ on $[a, b]$. For $0<$ $\varepsilon<(b-a) / 2, g_{k} \rightarrow g$ uniformly on $I_{\varepsilon}=[a+\varepsilon, b-\varepsilon]$, hence

$$
\|f-g\|_{I_{\varepsilon}}=\lim _{k \rightarrow \infty}\left\|f-g_{k}\right\|_{I_{\varepsilon}} \leq \lim _{k \rightarrow \infty}\left\|f-g_{k}\right\|=E(f)
$$


where $\|f-g\|_{I_{\varepsilon}}=\sup \left\{|f(x)-g(x)|: x \in I_{\varepsilon}\right\}$. Thus, as $g$ is $n$-convex, we have

$$
E(f) \leq\|f-g\|=\lim _{\varepsilon \downarrow 0}\|f-g\|_{I_{\varepsilon}} \leq E(f),
$$

so that $\|f-g\|=E(f)$, and the theorem is proved.

\section{REFERENCES}

1. R. B. Darst and S. Sahab, Approximation of continuous and quasi-continuous functions by monotone functions, J. Approx. Theory 38 (1983), 9-27.

2. R. Huotari and A. D. Meyerowitz, Best $L_{1}$-approximation by convex functions, preprint.

3. E. Kimchi and N. Richter-Dyn, 'Restricted derivatives' approximation to functions with derivatives outside the range, J. Math. Anal. Appl. 54 (1976), 786-798.

4. G. G. Lorentz and K. L. Zeller, Monotone approximation by algebraic polynomials, Trans. Amer. Math. Soc. 149 (1970), 1-18.

5. J. T. Marti, Konvexe analysis, Birkhäuser Verlag, Basel and Stuttgart, 1977.

6. A. W. Roberts and D. E. Varberg, Convex functions, Academic Press, New York, 1966.

7. H. L. Royden, Real analysis, 2nd ed., Macmillan, London, 1968.

8. D. Zwick, Properties of functions generalized convex with respect to a WT-system, J. Approx. Theory 33 (1981), 308-317.

Department of Mathematics, University of Vermont, Burlington, VerMONT 05405

Current address: Institut für Angewandte Mathematik der Universität Bonn, Wegelerstrasse 6, 5300 Bonn, West Germany 